\title{
Running speed in the dominance tube'
}

\section{JAMES F. BRENNAN, University of Dayton, Dayton, Ohio 45409}

Thirty-two male albino rats were divided into eight groups and run in the dominance tube according to a pairedcomparisons procedure. Two measures of speed were recorded. Overall speed was highly correlated with dominance. However, the number of times the dominant Ss progressed without interference from the submissive $S$ s was not highly correlated with dominance.

Schumsky \& Jones (1966) noted that the use of the dominance tube with rats may be criticized because of its measurement of only one dimension of dominance. A high correlation between speed and dominance would give even less credibility to the data obtained from the dominance tube because the winner could only be termed the faster animal, which may not agree with dominance as defined by another method, such as pellet competition as reported by Lester (1967). Attempting accurate measurement of speed, recent studies (e.g., Wilson, 1968; Dachowski, 1968) have employed photocells to activate timing devices to record speed and its relation to dominance. The purpose of this study was to investigate speed in the dominance tube utilizing a videotape unit which would permit more accurate data recording.

\section{METHOD}

Subjects were 32 male albino rats purchased from a professional supplier and housed singly. Ss were randomly assigned to eight groups and their tails were color coded for purposes of identification. Except for deprivation periods, food was available, and water was always available. Weights ranged from 293 to $312 \mathrm{~g}$ at the start of the experimental period.

Apparatus consisted of a dominance tube $\left(38 \times 2 \times 2 \frac{1}{2}\right.$ in.) which permitted room for only one $S$ to pass through at a time. At each end, separated from the tube by guillotine doors, were located goal-start boxes $\left(6 \times 4 \times 3 \frac{1}{2}\right.$ in.). One side of the tube was made of Plexiglas, permitting the filming of Ss while in the tube. A clock measuring in seconds and a scale divided into nine equal parts lying parallel to the tube were within camera range. Filming of each encounter was accomplished by an Ampex videotape recorder, Model VR-7000 with a Vikoa transistorized closed-circuit TV camera, Model ST-903.

Training consisted of placing each $S$ in the tube for $5 \mathrm{~min}$ on each of three successive days, so that they would be adapted to the tube. Following the 5 -min period on the third day, each group was placed on a 24-h food-deprivation schedule. The following day, each $S$ was individually run to a criterion of five runs from right to left allowing each $S 5 \mathrm{sec}$ in the goal box with a food pellet reward. On Day 5, each $S$ was similarly run five times from left to right, again allowing $5 \mathrm{sec}$ in the goal box. Ss were then fed ad lib for $24 \mathrm{~h}$, after which a deprivation of $24 \mathrm{~h}$ preparatory for the encounters began. On Day 8 , each group was run in a paired-comparison procedure of six encounters per group to establish a hierarchy. Each encounter was taped for later data analysis and timing was kept from the opening of the guillotine doors until the dominant $S$ had forced the submissive $S$ into its respective start box.

\section{RESULTS AND DISCUSSION}

Data was collected by analyzing the videotape and recording the number of wins for each $S$ in each group as a measure of dominance. Two measures of speed in the tube were taken. The first consisted of the number of times the dominant $S$ was faster over the entire encounter than the submissive $S$. The other measure of speed was determined by recording the number of times the dominant S's progress was slowed or stopped by the presence of the submissive $S$. Figures showing the position in the tube as a function of time in seconds were constructed showing the trend of the dominant $S$ in relation to the submissive $S$, in order to facilitate calculation of this second measure of speed. The results of the correlation $(r=.65)$ between the number of wins for each $S$ and the number of times the dominant $S$ was faster overall yielded significance $(p=.01)$. A second correlation was made between the number of wins for each $S$ and the number of times each dominant S's progress was not apparently slowed by the submissive $S$. This correlation $(r=.35)$ was not significant.

The results illustrated conflicting data from the two measures of speed and dominance employed. However, the first measure did not seem as credible as the second. The benefit of the use of the videotape for data collection allowed for a measure of speed and dominance which included the information of the first measure, while allowing observation of how speed affects dominance. While speed is involved in an overall appraisal of the dominance tube, the results of this study indicated that this speed factor is not strictly synonomous with dominance, adding to the defense of the dominance tube as an adequate measuring apparatus.

\section{REFERENCES}

DACHOWSKI, L. Reward size effects in the double runway. Psychonomic Science, 1968, 9, 157-158.

LESTER, D. Exploratory behavior of dominant and submissive rats. Psychonomic Science, 1967, 9, 285-286.

SCHUMSKY, D. A., \& JONES, P. D. Reliable paired comparison dominance in rats. Psychological Record, 1966, 16, 473-478.

WILSON; W. J. Adaptation to the dominance tube. Psychonomic Science, 1968, 10, 3, 119-120.

NOTE

1. The data presented in this paper was collected as part of a research project incorporated into a Master's thesis. The guidance of Prof. Joseph Rosa, Dr. Samuel Bower, and Dr. Lewis Hanes is gratefully acknowledged. 